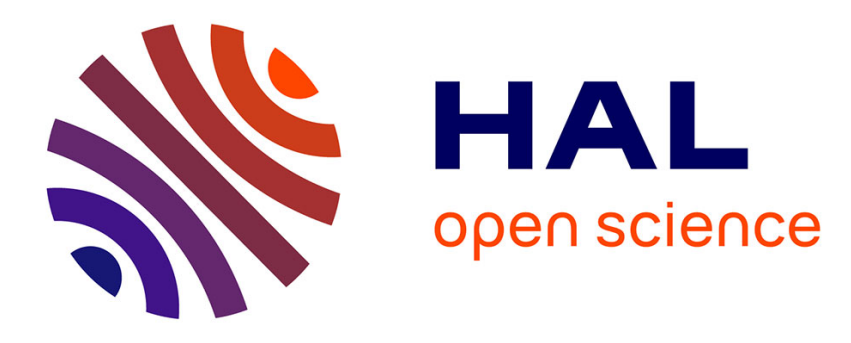

\title{
Local Trust Versus Global Trust Networks in Subjective Logic
}

Charif Haydar, Azim Roussanaly, Anne Boyer

\section{To cite this version:}

Charif Haydar, Azim Roussanaly, Anne Boyer. Local Trust Versus Global Trust Networks in Subjective Logic. Web Intelligence (WI) 2013, IEEE/WIC/ACM, Nov 2013, Atlanta, United States. pp.29-36, 10.1109/WI-IAT.2013.5 . hal-00929913

\section{HAL Id: hal-00929913 https://hal.inria.fr/hal-00929913}

Submitted on 14 Jan 2014

HAL is a multi-disciplinary open access archive for the deposit and dissemination of scientific research documents, whether they are published or not. The documents may come from teaching and research institutions in France or abroad, or from public or private research centers.
L'archive ouverte pluridisciplinaire HAL, est destinée au dépôt et à la diffusion de documents scientifiques de niveau recherche, publiés ou non, émanant des établissements d'enseignement et de recherche français ou étrangers, des laboratoires publics ou privés. 


\section{Local trust versus global trust networks in subjective logic}

\author{
Charif Haydar \\ Université de Lorraine \\ Laboratoire Loria, \\ Bâtiment C, Equipe KIWI \\ 615 , rue de jardin botanique \\ 54600 Vandœuvres-lès-Nancy, France. \\ Email: charif.alchiekhhaydar@loria.fr
}

\author{
Azim Roussanaly \\ Université de Lorraine \\ Laboratoire Loria, \\ Bâtiment C, Equipe KIWI \\ 615 , rue de jardin botanique \\ 54600 Vandœuvres-lès-Nancy, France. \\ Email: azim.roussanaly@loria.fr
}

\author{
Anne Boyer \\ Université de Lorraine \\ Laboratoire Loria, \\ Bâtiment C, Equipe KIWI \\ 615 , rue de jardin botanique \\ 54600 Vandœuvres-lès-Nancy, France. \\ Email: anne.boyer@loria.fr
}

\begin{abstract}
Social web permits users to acquire information from anonymous people around the world. This leads to a serious question about the trustworthiness of information and sources. During the last decade, numerous models were proposed to model social trust in the service of social web. Trust modeling follows two main axes; local trust (trust between pair of users), and global trust (user's reputation within the community).

Subjective logic, is an extension of probabilistic logic that deals with the cases of lack of evidences. An elaborated local trust model based on subjective logic already exists. The aim of this work is to apply this model to the first time on a real data set. Then, we propose another global trust model based also on subjective logic. We apply both models on a real data set of a question answering social network that aims to assist people to find solutions to their technical problems in various domains. Our proposed global trust model ensures a better performance thanks to its precise interpretation of the context of trust, and its ability to satisfy new arrived users.
\end{abstract}

\section{INTRODUCTION}

Web 1.0 provided a popular access to the largest data store ever existed (Internet). The major difficulty resided in extracting relevant information and resources from the huge mass of data available for most queries. Information retrieval (IR) came out to yield Internet more efficient and exploitable by ranking resources according to their relevance to queries. Then, web 2.0 arrived with more interactive tools such as forums and social networks. The numerous people who were only the spectators in web 1.0, became the actors in web 2.0. They are now able to share their own opinions and knowledge. Collaborative IR and social recommender systems (RS) [30] are now used to rank this kind of resources.

Practically, social web permits users to acquire information from anonymous people around the world. This leads to a serious question about the trustworthiness of information and sources. To get over this problem, many computational models were proposed to model social trust and integrate it in different social online domains such as social networks, recommender systems [7], [18], file sharing [25] ...etc.

Social trust, for us, is the belief of an individual, so called truster, that another individual, so called trustee, has the competence and the willingness to execute or to assist in the execution of a task to the favor of the truster. The assistance can simply consist in recommending another individual to execute the task. The truster tries to acquire information and construct his own belief about the trustee before deciding to cooperate with him. We are interested in two methods to construct the belief about the trustee; the first is called local trust, it depends on the past interactions between the truster and the trustee, and on the word of mouth mechanism that allow the truster to acquire information about the trustee from his friends and theirs'. The second is called global trust, it depends on the reputation of the trustee within the community.

Subjective logic (SL) [29] is an extension of probabilistic logic, based on the belief theory [3], [2]. SL was already used to build a local trust model (LTSL) [5]. To the best of our knowledge, this model was never experimented with real data. In this paper, we propose a new global trust model based on subjective logic (GTSL), we implement the local trust model and compare the performance of both models on real data, and we compare them to a referential trust model called Moletrust [7].

The object of our application is the dataset stackoverflow ${ }^{1}$. It is a social website based on a question answering platform to assist users to find answers to their questions in divers domains (programming, mathematics, English language, cooking ...). We assume that proposing an answer is a proof of willingness to assist the asker. Therefore, our objective is to find the user capable to provide the most relevant answer in function to his competence in the current context.

Local trust models suffer from a cold start problem, they can not deal neither with new users nor with users having no friends [32]. Global trust models are not concerned by this problem. It is efficient even to cold start users, which improves its performance.

All three models in this paper are context aware, still their interpretations of the context are distinct. LTSL and Moletrust consider the domain of the question as the context, whereas GTSL considers a more refined context relying on the subject of the question. The precision in defining the context is another key element to GTSL to surpass the performance of the other two models.

\footnotetext{
${ }^{1}$ http://www.stackoverflow.com
} 
The paper is organized as following: in section II we present the general framework, starting by presenting social trust and computational trust. In II-B we introduce subjective logic and some of it's operators. In section III, we describe the local trust SL model that we implement, and the global trust SL model that we propose. Section IV is dedicated to argue about other trust models and justify our choice of SL. In section $\mathrm{V}$, we describe the used dataset, and present our evaluation method. In section VI we discuss our results. Finally in section VII we present our conclusions and future work.

\section{GENERAL FRAMEWORK}

The objective of trust is to find the appropriate person to cooperate with in order to achieve a given task or context. Truster's decision about to cooperate or not is influenced by many factors. In the following section we present a real life example about trust in order to explain this phenomena, and some factors that can influence the cooperation decision.

Suppose that Alice needs to repair her car. Alice knows Bob and Eric. Alice trusts Bob, but he has no experience in repairing cars. Even though Alice trusts Bob, she will not ask him to repair her car because she thinks that he lakes competence. Eric is mechanician, but the last time he changed her car's water pump he affixed a second hand one, and charged her for a new one. Alice considers Eric dishonest, so unwilling to execute the task properly. Nevertheless, Alice might accept to cooperate with Eric in certain extreme cases; for example if she is stranded nearby to his garage, or if no other mechanician is available.

Alice uses her own experience and past interactions with Bob and Eric to decide if she will cooperate with one of them or not. This example show the major role of the Alice's belief about the competence and willingness of Bob and Eric. The context here is (vehicle repairing). Hence, the belief of Alice is related to the context (basically Bob is trustee, but not in this context). In other words, Alice's decision is related to her past interactions with Bob and Eric, and the current context.

Suppose now that Bob knows another mechanician called Dave. Bob was always satisfied by the work of Dave to repair his car. Bob recommends Dave to Alice. Alice accepts to cooperate with Dave although she does not know him personally, just because he is recommended by a trustee friend. Although trust is not transitive (in the mathematical meaning of transitivity), but it is still influenceable by intermediate persons [5], [27], [7], [18]. If no past direct interaction between the truster and the trustee, a trustee intermediate can assist to establish a link between them at a first time, the link then will be updated by direct interactions, so if Alice was satisfied by the work of Dave, her trust in him will increase, otherwise it will decrease. This is how the word of mouth works. Trustee friends can assist in accomplishing the task by recommending other people. Hence, trust relationships might have different types and purposes. In [5], authors define four properties of trust relationships:

1) Direct trust: trust is the result of interactions between exclusively the truster and trustee, such as the relations Alice Bob, Alice Eric and Bob Dave.
2) Indirect trust: the two persons do not know each others. Trust is established due to trustee intermediate persons, such as the relation Alice Dave.

3) Functional trust: the expectation of the truster is that the trustee accomplish the task himself, such as the relation Alice Eric and Alice Dave.

4) Referential trust: the expectation of the truster is that the trustee will recommend someone to accomplish the task, such as the relation Alice Bob. Note that recommendation of Bob could also be based on his referential trust in another person who knows Dave. In other words, no obligation for the trustee in referential trust to base his recommendation on a functional trust relation. Normally a series of referential trust relations must end with one functional trust relation [11].

Alice may receive advices from other people, such as in figure 1, where continuous lines represent trust, and dashed ones represent distrust. Alice has contradictory information about Dave. Treating these information is a complicated issue. For example, while Alice distrusts Fred, should she neglect his opinion or consider its opposite?

If Alice were new in the city, and has no friends yet. The word of mouth technique would not be useful for her. Nevertheless, she can rely on the reputation of Dave using for example a specialized magazine in classifying mechanisians, or even by contacting the manufacturer of her car and asking for a certified mechanisian in her zone. Till now we considered the context as "vehicle repairing", we could be more precise by including for example the mark, the model and the fabrication year of the vehicle. Dave who used to be a good mechanic for Bob's car, might be less competent for Alice's if it is very different from Bob's car. Refining the context can lead to a more precise search [33].

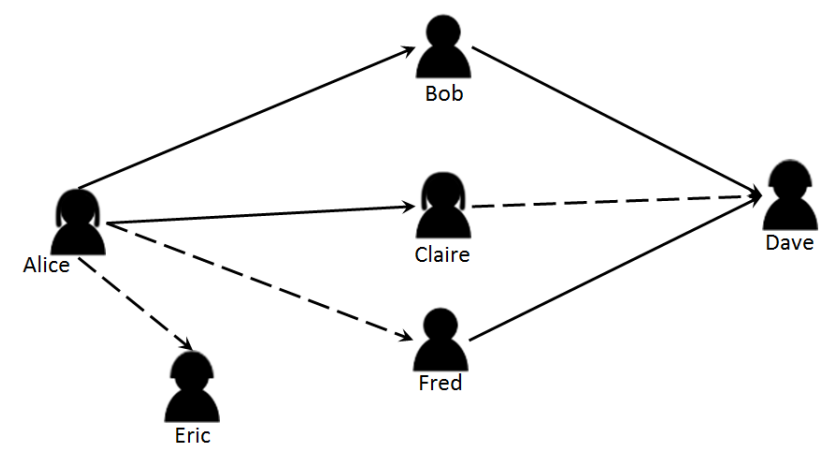

Fig. 1. Trust network

This example shows how the truster can construct his belief about the trustee using his past interactions and the word of mouth technique. It shows also the limit of this method for new users, and how a reputation based system can make more information available to them if they have no or less friends. Moreover, it demonstrate the important role of the context and the impact of its interpretation.

In the next section we discuss the formalization of social trust for the social web. 


\section{A. Computational trust}

Computational trust is the formalization of social trust for computer science and its applications. It raised in the last decade to satisfy the needs of trust awareness in online applications. Computation trust has three axes [15]:

- Quantitative, also called global-trust or Reputation: where the system computes a score for each user, this score represents his global trustworthiness. This same score is considered when any other user needs to interact with this user [16].

- Qualitative, also called local-trust or relationship: it takes into account the personal bias. Represented as user to user relationship. It is the trustworthiness of a user $\mathrm{Y}$ from the point of view of only one user X. Some researchers call this local-trust [16].

- Process driven (system): it represents the trust of the users in the system. This kind of trust is not considered in this paper.

Many models were proposed [12], [13], [14] to model trust, they tend to formulate local trust problem within a trust network. A trust network is a directed weighted graph where vertices represent users and edges represent trust relationships. Models differentiate by their notation of edges and their strategies in traversing the network to compute trust between unconnected people, this operation is called trust propagation. As for global trust, it is usually presented by associating each user by a score that implies his reputation [25], [26].

Subjective logic (SL) is a probabilistic model that was used to model local trust in [5]. It has many operators that allow it to integrate many aspects and factors of trust. In this work we use SL local trust model, and then we propose a new SL global trust model. The following section II-B is dedicated to explain the structure and some operators of subjective logic.

\section{B. Subjective logic}

Subjective logic (SL) [29] is an extension of probabilistic logic, which associates each probability with a degree of uncertainty. Subjective logic allows to build models that treat with situations of incomplete evidences.

Belief theory [3], [2] is a special case of probability theory dedicated to treat incomplete knowledge. The sum of probabilities of possible cases can be less than 1 . Subjective logic [4] offers a belief calculus using a belief metrics called opinion. The opinion of an individual $U$ about a statement $x$ is denoted by:

$$
\omega_{x}^{U}=(b, d, u, a)
$$

Where: $b, d, u \in[0,1]$ are respectively the belief, disbelief and uncertainty of $U$ about $x$. The sum of the three values equals to one (i.e $b+d+u=1$ ). Base rate $a \in[0,1]$ is the prior probability. Basically, base rate is a statistical measure applied in cases of evidences' absence. For example, when we know that the percentage of a disease $x$ in a given population is $1 \%$, then the base rate of $x$ 's infection is $1 \%$. When we meet a new individual who did not make a test for the disease, priori we assume that the probability that he is infected is $1 \%$. In social trust case, while no priori statistics are present, we consider that unknown person has a half chance to be trustworthy. So we use a base rate $a=0.5$. In subjective logic, the base rate steers the contribution of the uncertainty in the computation of the probability expectation value according to the equation 1 :

$$
E\left(\omega_{x}^{U}\right)=b+a \times u
$$

The opinion in subjective logic is based on the accumulation of successful and failed experiences. After each experience, $U$ updates his opinion about $x$ consistently with experience's outcome. According to this description, opinion can be represented as a binary random variable. Beta distribution is normally used to model the behavior of this kind of variables. By consequence, the opinion corresponds to the probability density function (PDF) of beta distribution. PDF is denoted by two evidence parameters $\alpha$ and $\beta$ that can be written as functions of the number of successful and failed experiences respectively.

$$
\begin{aligned}
& \alpha=r+W \times a \\
& \beta=s+W \times(1-a)
\end{aligned}
$$

Where $r$ is the number of successful experiences (evidences). $s$ is the number of failed experiences. $W$ is the noninformative prior weight which ensures that the prior (i.e. when $\mathrm{r}=\mathrm{s}=0$ ) Beta PDF with default base rate $\mathrm{a}=0.5$ is a uniform PDF (normally $W=2$ ).

The expectation value of beta PDF is:

$$
E(\operatorname{Beta}(p \mid \alpha, \beta))=\frac{\alpha}{\alpha+\beta}=\frac{r+W a}{r+s+W}
$$

In subjective logic, the mapping between the opinion parameters and the beta PDF parameters is given as follow:

$$
\begin{aligned}
& b=\frac{r}{(r+s+W)} \\
& d=\frac{s}{(r+s+W)} \\
& u=\frac{W}{(r+s+W)}
\end{aligned}
$$

Table I shows an example of the evolution of an opinion with successive interactions.

\begin{tabular}{|c|r|c|c|c|c|c|}
\hline No & state & r & s & belief & disbelief & uncertainty \\
\hline 0 & no interaction & 0 & 0 & 0 & 0 & 1 \\
\hline 1 & successful interaction & 1 & 0 & $1 / 3$ & 0 & $2 / 3$ \\
\hline 2 & failed interaction & 1 & 1 & $1 / 4$ & $1 / 4$ & $2 / 4$ \\
\hline 3 & successful interaction & 2 & 1 & $2 / 5$ & $1 / 5$ & $2 / 5$ \\
\hline
\end{tabular}

In the first line of table I we see the case of absence of evidences (experiences). The opinion is completely uncertain $(u=1)$. In this case, according to equation 1 , the expectation value equals to the base rate value. The arrival of new experiences, will cause that the uncertainty decreases, regardless if 
these experiences are successful or failed. Successful experiences will augment the belief, whereas failed experiences will augment the disbelief.

Subjective logic opinions can be illustrated in the interior of an equal-sided triangle. The three vertices of the triangle are called belief, disbelief, and uncertainty. The uncertainty axis links the uncertainty vertex with the opposite edge (the beliefdisbelief edge), the uncertainty value of the opinion is plotted on this axis considering that its contact with the edge beliefdisbelief represents the value 0 , whereas the contact with the uncertainty vertex represents the value 1 . In the same way we describe the belief and the disbelief axis.

The opinion is represented by the intersection point of the three projections on the three axis (belief, disbelief and certainty) is shown in the example in figure 2 . The bottom of the triangle is the probability axis, the probability expectation value is the projection of the opinion point on the probability axis with respect to the line linking the uncertainty vertex with the base rate point on the probability axis. Figure 2 illustrates an example of opinion mapping in subjective logic. The opinion is represented by a point inside the triangle. The point is the intersection of the projection of the three values $b, d$, and $u$ on the axis of belief disbelief and uncertainty respectively. the probability expectation value $E(x)$ is the projection of $\omega_{x}$ on the probability axis directed by the axis linking $a_{x}$ with the uncertainty edge.

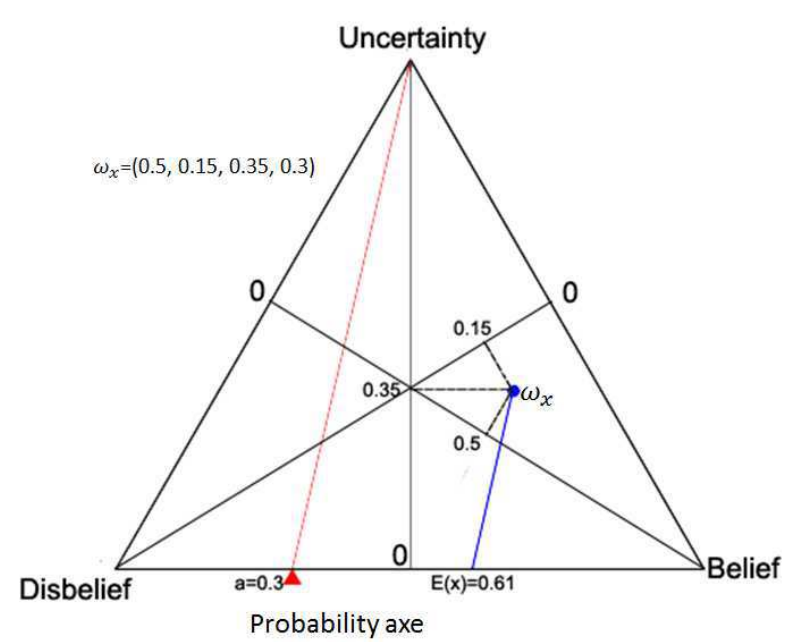

Fig. 2. Opinion

Note that changing the value of base rate can make people more reckless or more cautious.

After defining the structure of the opinion in subjective logic, we need to explain some of subjective logic operators that are useful for building trust network. Local trust networks are usually represented by a direct graph, where vertices represent users, and edges represent trust relations. By consequence, computing trust value between two users is reduced to finding a path or more connecting them to each other.

1) Trust transitivity: If an individual $A$ trusts another individual $B$, and $B$ trusts $C$, trust transitivity operator is used to derive the relation between $A$ and $C$. Almost all trust algorithms propose transitivity operators. In [8], the inferred value is restrained by the value of trust between $A$ and $B$. In [7], it is inversely proportional to the distance between $A$ and C.

Although trust transitivity has no mathematical evidences, but users generally tend to trust people who are trusted by their friends. Building a trust transitivity operator is much easier in "trust only" models. The complicated issue is when $A$ distrusts $B$. In this case, $A$ can has one over two reactions: 1) $A$ can consider that $B$ has bad intentions towards him, so he will behave to the opposite of the opinion of $B$. 2) $A$ can consider that $B$ is just inept to argue about $C$, so he will neglect his opinion.

Subjective logic proposes two transitivity operators corresponding to two reactions, they are by order: Opposite belief favouring operator and uncertainty favouring operator.

Assume that the opinion of $A$ about $B$ and the opinion of $B$ about $C$ are given as follows:

$$
\begin{aligned}
& \omega_{B}^{A}=\left(b_{B}^{A}, d_{B}^{A}, u_{B}^{A}, a_{B}^{A}\right) \\
& \omega_{C}^{B}=\left(b_{C}^{B}, d_{C}^{B}, u_{C}^{B}, a_{C}^{B}\right)
\end{aligned}
$$

Then the trust of $A$ in $C$ can be inferred by the opposite belief favouring operator as follows:

$$
\omega_{B}^{A} \otimes \omega_{C}^{B}=\left\{\begin{array}{l}
b_{C}^{A: B}=b_{B}^{A} \cdot b_{C}^{B}+d_{B}^{A} \cdot d_{C}^{B} \\
d_{C}^{A}: B=b_{B}^{A} \cdot d_{C}^{B}+d_{B}^{A} \cdot b_{C}^{B} \\
u_{C}^{A}: B=u_{B}^{A}+\left(b_{B}^{A}+d_{B}^{A}\right) \cdot u_{C}^{B} \\
a_{C}^{A: B}=a_{C}^{B}
\end{array}\right.
$$

or by the uncertainty favouring operator:

$$
\omega_{B}^{A} \otimes \omega_{C}^{B}=\left\{\begin{array}{l}
b_{C}^{A: B}=b_{B}^{A} \cdot b_{C}^{B} \\
d_{C}^{A: B}=b_{B}^{A} \cdot d_{C}^{B} \\
u_{C}^{A: B}=d_{B}^{A}+u_{B}^{A}+b_{B}^{A} \cdot u_{C}^{B} \\
a_{C}^{A: B}=a_{C}^{B}
\end{array}\right.
$$

In this work, while the used dataset a priori does not contain attackers or malicious users, we consider that distrust relations are mostly driven by incompetence rather than bad intentions, that is why in the rest of this paper we use uncertainty favouring operator for trust transitivity.

2) Opinion fusion: Suppose in the previous example that $A$ has another trustee friend $D$ how also trusts $C$. $A$ has two separate sources of information about $C$.

This operator allows to aggregate the opinions of two users ( $B$ and $D$ ) about a third user $C$. Its idea is based on cumulating evidences about $C$ from both $B$ and $D$.

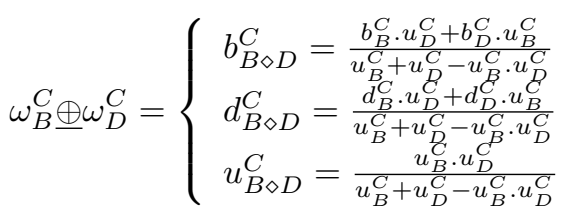

Even if the opinions of $B$ and $D$ were contradictory, the operator can still cumulate their evidences to generate an unique opinion based on them. 


\section{PROPOSED Models}

The aim of our models is to predict the most relevant answer to a given question within a list of answers. We employ two types of trust aware models that propose two different interpretation of context. The first (LTSL) is based on the local trust, and the second (GTSL) is based on the global trust. Both models use subjective logic.

\section{A. Local trust subjective logic (LTSL)}

This model is originally proposed in [5]. To the best of our knowledge it was never applied to real data. It consists in building a local trust network between users. The edges of this network are SL opinions of users about each other. Whenever a user $A$ accepts the answer of user $B$ to his question, the opinion of $A$ in $B$ is updated by adding a new successful interaction between them

In stackoverflow, $A$ can accept only one reply out of the list of available answers. Unaccepted answers are not necessarily bad ones. They might be simply not good enough compared to the accepted one. They even might be better but arrived too late that $A$ has already accepted another satisfactory answer. Basically, while we do not have an explicit reaction from $A$ towards the unaccepted answers, we suppose four hypotheses to treat them:

1) rigorous hypothesis: unaccepted answers are considered as failed interactions.

2) ignoring hypothesis: unaccepted answers are not considered at all.

3) independent subjective hypothesis: In both precedent methods, the interaction value is either +1 (successful), or -1 (failed). In this method we introduce relatively successful/failed interactions. We use the rates of community towards the answer to estimate a subjective successful/failure of the interaction. In fact, the thumb-up represents a successful interaction with an unknown user, same thing for the thumb-down with a failed interaction. The global reaction of the community towards the answer is subjective opinion resulting from members' interactions with the answer. We consider the expectation value of the community's opinion as the value of the partially successful/failure of the interaction between the asker and the replier.

4) dependent subjective hypothesis: regarding to the fact that a user can give a thumb-up for an answers because it is better/worse than others, the attribution of thumb-up and thumb-down can be relative too. The reason why we propose another subjective method where our certainty is influenced by the global number of thumb-up and thumb-down attributed to all answers of the same question. In this case the opinion about an answer is dependent on the the other opinions about the other answers.

$$
\text { Certainty }_{j}=\frac{\sum_{j} t h}{2+\sum_{i=a n_{0}}^{a n_{n}} \sum_{i} t h}
$$

Where $t h$ is an absolute value of thumb (up or down). $j$ is the current answer.

$n$ is the number of answers of the current question.
$\mathrm{U}+$ ncertainty is the complement of certainty to 1 :

$$
\text { uncertainty }_{j}=1-\text { certainty }_{j}
$$

The default non-informative prior weight $\mathrm{W}$ is normally defined as $\mathrm{W}=2$ because it produces a uniform Beta PDF in case of default base rate $\mathrm{a}=1 / 2$. The three components of the opinion are:

$$
\text { belief }_{j}=\text { uncertainty }_{j} \times \frac{\sum_{j} t h_{u p}}{\sum_{j} t h}
$$

Where $\sum_{j} t h_{u p}$ is the number of thumbs up attributed to the answer.

$$
\text { disbelief }_{j}=\text { certainty }_{j} \times \frac{\sum_{j} t h_{\text {down }}}{\sum_{j} t h}
$$

Where $\sum_{j} t h_{\text {down }}$ is the number of thumbs down attributed to the answer.

Finally the computes the expectation value of the resulting opinion and consider it as the value of the relative success/failure interaction.

To predict the accepted answer of a given question $Q$ asked by the user $A$, we identify $R$ the set of users who contributed answers to the current question. Then, we traverse the graph (trust network) to compute the local trust between the owner of the question and each of them. We assume that $A$ will accept the answer of the most trustee user within $R$.

\section{B. Contextual global trust model (GTSL)}

Each question in stackoverflow has a set of associated keywords. We use these keywords to build a new global trust model. that considers the reputation of users towards keywords. When a user $A$ accepts the answer of a user $B$ to his question, a link is created or updated between $B$ and each one of the keywords associated to the question. As for unaccepted answers we apply the same four hypothesis in III-A. The semantic signification of the links between users and keywords is the reputation of the user towards the keyword. A reputed user towards a keyword can also be called expert.

To predict the accepted answer of a given question $Q$ asked by the user $A$, we identify $R$ the set of users who contributed answers to the current question, and the set $K$ of keywords associated to the question. We compute the average reputation score to each member of $R$ towards the elements of $K$. The member with the highest average score is chosen to be the owner of the accepted answer.

\section{RELATED WORK}

Computational trust is applied to many fields in artificial intelligence, recommender systems, file sharing, Spam detection, networks security ...etc. Most computational models are not completely loyal to the social trust, they are fitted to their application fields and context. Our choice of the trust model (SL) is motivated by its high faithfulness to the social trust. To the best of our knowledge, SL is usually employed in local trust networks, but its flexibility allowed us to build our contextual global trust model. In addition to its capacity to work in environment with lack of evidences like ours. 
In [25] a global trust model used to improve the confidence in a file sharing network, it considers trust as a limited quantity by person, so the more he trusts people the less he can attribute trust to each of them. Another older model in [26] used to detect malicious attacks in a developers social network, it uses a global trust metrics which can not be contextualized, and is not prepared to evidences' lack environment. Appleseed [16], tidaltrust [18] and Moletrust [7] are local trust models. They are applied in recommendation contexts. They deal only with positive values of trust, so they do not take the distrust into account. Moletrust and tidaltrust employ different methods to traverse the graph between a source user (truster) and a destination user (trustee). They look for the shortest path that maximizes the trust between the two users. Subjective logic proposes a different strategy, it allows all truster's friends to contribute their opinions about the destination, and then aggregates these opinions regarding the certainty of each friend towards his opinion. If a friend of the truster has no opinion towards the trustee, he will ask his friends in turn and so on. So the most important measure in SL is not the distance from the truster but the certainty of opinion.

Model in [20] is used to enhance the relevance of information on the web but also using only positive trust. Other models that consider only trust exist in [21], [22], [23], [24].

Authors in [19] proposes a model based on enhancing the collaborative filtering algorithm by associating a global trust score to each user, the score is based on the success of the recommendations in which he was involved. This score represents the usability of the user for the system.

Subjective logic considers trust and distrust relationships. Trust relationships in SL are based on accumulation interactions. It is able to aggregate the recommendation of many friends. It possesses numerous operators that allow it to be adaptable for different visions about trust.

Although most definitions of social trust accord the importance of the context, in most model, context is missed during the conception, or integrated as a peripheral element. In the following section we compare the performance of local trust subjective logic network to a reference network based on a shortest path method (Moletrust), then we compare them with the contextual global trust model.

\section{EXPERIMENTAL WORK}

We use the dataset of the website stackoverflow. The website offers a question answering forums for multiple domains, mainly but not limited to computer science. The available data contains 30 domains. Users subscribe to the website by domain, so one user can have multiple accounts, according to the number of domains in which he participates. The total number of accounts is 374,008 for about 153,000 users.

User asks a question in a given domain, and associates a set of keywords to his question, then he receives many answers. He chooses the most relevant answer to him and attributes an "accepted answer" label to it. Nevertheless, users can keep proposing new answers. Subsequent users who have the same problem as the asker can profit of answers and rate them on their usefulness by attributing thumb-up or thumb-down. In the available dataset, we have access to only the total number of thumbs-up and total number of thumbs-down an answer has, but no information about suppliers' identities. The website, offers the possibility to order answers by relevance, where the accepted answer is put in the top of the list, followed by the other answers ordered by the difference between thumbs-up count and thumbs-down count. Our work aims to use trust based models to predict the accepted answer over the set of available answers. Total number of questions in current dataset equals to 371,594 , for a total number of answers 816,487 . We divide the questions of each domain in five equivalent sets. Then, we apply a crossing test in five iterations, in each iteration we use four sets for learning and building the trust network and the fifth for testing the prediction quality.

\section{A. Evaluation}

While both proposed methods are based on subjective logic, we would like to compare them to a referential trust method. To the opposite to its matches, Moletrust algorithm considers the expression of trust as a binary value, and user can only expresses positive trust. This is compatible with the explicit part of the dataset, while the users can only accept answers by binary positive notation. Moletrust is a shortest path trust method, showed efficiency in another application context [31], [32]. We use it in our application as a base reference to evaluate the performance of SL methods.

1) Moletrust: Moletrust was presented in \{massa04. It considers that each user has a domain of trust, where he adds his trustee friends to. User can either fully trust other user or not trust him at all. The model considers that trust is partially transitive, so its value decline according to the distance between the source user and the destination user. The only initializing parameter is the maximal propagation distance $d$.

If user $A$ added user $B$ to his domain, and $B$ added $C$, then the trust of $A$ in $C$ is given by the equation:

$$
\operatorname{Tr}(A, C)=\left\{\begin{array}{ll}
\frac{(d-n+1)}{d} & \text { if } n \leq d \\
0 & \text { if } n>d
\end{array}(10)\right.
$$

Where $n$ is the distance between $A$ and $C(n=2$ as there two steps between them; first step from $A$ to $B$, and the second from $B$ to $C$ ).

$d$ is the maximal propagation distance.

$$
\text { Consider } d=4 \text { then: } \operatorname{Tr}(A, C)=(4-2+1) / 4=0.75 \text {. }
$$

We consider that when a user $A$ accepts an answer of another user $B$, that $A$ trust $B$. A Moletrust link between both users is created. While the algorithm is not aware to distrust so no interpretation exists for unaccepted answers.

2) Evaluation Metrics: We regard the problem of finding the accepted answer as a list ranking problem with one relevant item. Mean reciprocal rank (MRR) is a quality metrics used to evaluate systems that have to give out a ranked list with only one relevant item. Reciprocal rank (RR) of question is $1 / r$ where $r$ is the rank given by the evaluated algorithm to the accepted answer. Mean reciprocal rank is the mean value of RR's to all questions. The value of this metrics varies between 0 and 1 , where 1 is the best precision score. 
MRR is a good indicator to the performance of prediction algorithms for ranked lists. Nevertheless, we think that it is not perfectly adapted to our case. MRR is usually employed for systems that have to predict a list of items within the which a relevant item exists. We are trying to find the accepted answer by reranking an existing list of answers. Remark the case when the algorithm ranks the relevant item in the last position of the list, the algorithm is recompensed for at least having chosen the item within the list. In our case the list predefined, so the algorithm should not be recompensed for ranking the relevant item at the end of the list. The range of $\mathrm{RR}$ values is $[1 / r, 1]$, we propose a modified version where the value varies between 1 if the relevant item is in the top of the list, and 0 if it is at the end of the list. We call this metrics mean predefined lists rank(MPLR), where PLR is given by the formula:

$$
P L R=\frac{N-r}{N-1}
$$

Where: $N$ is the size of the list.

We employ a modified competition ranking strategy, so the ranking gap is left before equal items. Example if two items on the top of the list have the same score, the are considered both second, and no item is put in the first rank.

\section{RESULTS AND DISCUSSIONS}

Over the 371,594 available questions in the corpus only 118,778 have more than one answer and have an accepted answers. Only these questions are suitable to apply our test.

Table II illustrates the MRR scores of the three models, and table III illustrates MPLR scores. MPLR scores are lower than those of MRR. Nevertheless both tables lead to the same conclusions.

Obviously, subjective logic models surpass Moletrust when applied in the same conditions (ignoring hypothesis). This is in the first place because the power of the probabilistic base of SL, and its capacity to accumulate interactions, whereas Moletrust considers one interaction sufficient to establish an absolute trust relation. In the second place this shows that the strategy of shortest path is not always the efficient one. Even though, the flexibility of subjective logic allowed to slightly improve the scores by using the three other hypothesis. Local trust models increases its score about $4 \%$ thanks to the probabilistic hypothises, and the global trust models increases between $7 \%$ and $14 \%$ with other hypothises.

It is also obvious that GTSL surpasses widely LTSL. LTSL suffers from a cold start problem, it is unable to predict answers to users who has no or less friends. In current dataset, about $20 \%$ of profiles $(74,163$ over 374,008$)$, have asked exactly one question each. $14 \%$ of the 118,778 questions included in our test are generated by these profiles. LTSL can not predict answers to questions generated by such isolated profiles. GTSL can predict answers to these users. Basically, the truster in LTSL builds his belief by exploiting his own interactions, and those of his friends and their friends. GLSL offers a larger archive of interactions to the trusters. Truster in GLSL can has access to all the past interactions of the trustee, so construct a more elaborated belief about him.
Local trust SL model considers the domain of the question as a context. Global trust model considers a more refined interpretation of the context, based on a sub-domain defined by the tags associated to the question. The difference in both models scores shows that trust is very sensible to the context. The context in global trust model is very adaptive, this leads to a more specific person having competences in this exact context. The presence of this person in the list of people who answered the question proves his willingness to assist the asker, his competence and mastery of subject lead him to be the owner of the accepted answer. Malicious attackers who might supply answers for bad intentions will be automatically rejected or classed in the bottom of the list, because of their weak global trust score. On the other hand, context in the local trust model is more fuzzy. In real life if $B$ was able to answer a question of $A$ about Java programming language, this does not mean that he would be able the next time to reply to a question about $\mathrm{C}++$ programming language, although it is still the same domain (context) for the local trust model. So even within the same domain, people might be experts in narrow sub-domains, while having a general or even weak knowledge about the other parts of the domain.

Our results stand up for a superiority of global trust model and its interpretation of the context, and a superiority of subjective logic models over Moletrust. We mentioned that SL models improve their scores by means of the hypothesis to interpret the implicit data, but both models are influenced differently by those hypothesis. In local trust model, the probabilistic hypothesis slightly surpass the score of the other hypothesis, because they enrich the trust network by more interactions between users. On the other hand, the global trust model achieves its best score with the rigorous hypothesis because it allows to be more strict while appointing the experts.

\begin{tabular}{|c|r|c|c|}
\hline method & MoleTrust & Local trust SL & Global trust SL \\
\hline Rigorous & - & 0.57 & 0.884 \\
\hline Ignoring & 0.53 & 0.58 & 0.7 \\
\hline Dependent probabilistic & - & 0.62 & 0.815 \\
\hline Independent probabilistic & - & 0.617 & 0.78 \\
\hline
\end{tabular}

\begin{tabular}{|c|r|c|c|}
\hline method & MoleTrust & Local trust SL & Global trust SL \\
\hline Rigorous & - & 0.37 & 0.85 \\
\hline Ignoring & 0.3 & 0.36 & 0.6 \\
\hline Dependent probabilistic & - & 0.442 & 0.76 \\
\hline Independent probabilistic & - & 0.438 & 0.73 \\
\hline
\end{tabular}

TABLE III. MPLR RESULTS

\section{CONCLUSION AND FUTURE WORKS}

As we argued in the beginning, trust is affected by many factors including context. This work shows the efficiency and the adaptability of subjective logic, but it is also dedicated to explain the influence of the context on trust models' performance. We would like to make use of the flexibility of SL to study other factors that affect the trust such as the temporality: does a recent interaction has the same importance as an old one?. Our actual models consider only direct interactions, other incremental models update links after each successful/failed recommendation. We would like to study the impact of these recommendation interactions. 


\section{REFERENCES}

[1] R. Burke. 2007. Hybrid web recommender systems. In The adaptive web, Peter Brusilovsky, Alfred Kobsa, and Wolfgang Nejdl (Eds.). Lecture Notes In Computer Science, Vol. 4321. Springer-Verlag, Berlin, Heidelberg 377-408.

[2] Yager, Ronald R., Janusz Kacprzyk, and Mario Fedrizzi, eds. Advances in the Dempster-Shafer Theory of Evidence. New York, NY, USA: John Wiley amp; Sons, Inc., 1994.

[3] Dempster, A.P. The DempsterShafer Calculus for Statisticians. International Journal of Approximate Reasoning 48, no. 2 (juin 2008): 365377. doi:10.1016/j.ijar.2007.03.004.

[4] Josang, A. A Logic for Uncertain Probabilities. International Journal of Uncertainty, Fuzziness and Knowledge-Based Systems 9, no. 03 (2001): 279311.

[5] Josang, A., R. Hayward, and S. Pope.Trust Network Analysis with Subjective Logic. In Proceedings of the 29th Australasian Computer Science Conference-Volume 48, 8594, 2006. http://dl.acm.org/citation.cfm?id=1151710.

[6] Josang, Audun. The Consensus Operator for Combining Beliefs. ARTIFICIAL INTELLIGENCE JOURNAL 142 (2002): 157170.

[7] P. Massa and B. Bhattacharjee. Using trust in recommender systems: An experimental analysis. In iTrust04, pages 221235, 2004.

[8] Jennifer Golbeck. Personalizing applications through integration of inferred trust values in semantic web-based social networks. In Semantic Network Analysis Workshop at the 4th International Semantic Web Conference, November 2005.

[9] Marsh, Stephen, and Pamela Briggs. Examining Trust, Forgiveness and Regret as Computational Concepts. In Computing with Social Trust, [3 edited by Jennifer Golbeck, 943. HumanComputer Interaction Series. Springer London, 2009.

[10] Josang, A., E. Gray, and M. Kinateder. Simplification and Analysis of Transitive Trust Networks. Web Intelligence and Agent Systems 4, no. [3 2 (2006): 139161

[11] Josang, A., and S. Pope. Semantic Constraints for Trust Transitivity. In Proceedings of the 2nd Asia-Pacific Conference on Conceptual[32] modelling-Volume 43, 5968, 2005.

[12] Abdul-Rahman, A., and S. Hailes. Supporting Trust in Virtual Communities. In Proceedings of the 33rd Annual Hawaii International Conference on System Sciences, 2000, 9 pp. vol.1, 2000.[33] doi:10.1109/HICSS.2000.926814.

[13] Krukow, Karl. Towards a Theory of Trust for the Global Ubiquitous Computer: A Dissertation Presented to the Faculty of Science of the University of Aarhus in Partial Fulfilment of the Requirements for the $\mathrm{PhD}$ Degree. Department of Computer Science, University of Aarhus, 2006.

[14] Mui, L. Computational Models of Trust and Reputation: Agents, Evolutionary Games, and Social Networks. PhD Thesis, Massachusetts Institute of Technology, 2002.

[15] Kwan, Ming, and Deepak Ramachandran. Trust and Online Reputation Systems. In Computing with Social Trust, edited by Jennifer Golbeck, 287311. HumanComputer Interaction Series. Springer London, 2009.

[16] Ziegler, C. N., and G. Lausen. Spreading Activation Models for Trust Propagation. In e-Technology, e-Commerce and e-Service, 2004. EEE04. 2004 IEEE International Conference On, 8397, 2004.

[17] Paul Resnick and Hal R. Varian. 1997. Recommender systems. Commun. ACM 40, 3 (March 1997), 56-58.

[18] Golbeck, Jennifer Ann. Computing and Applying Trust in Web-based Social Networks, 2005.

[19] ODonovan, J., and B. Smyth. Trust in Recommender Systems. In Proceedings of the 10th International Conference on Intelligent User Interfaces, 167174, 2005. http://dl.acm.org/citation.cfm?id=1040870.

[20] Richardson, Matthew, Rakesh Agrawal, and Pedro Domingos. Trust Management for the Semantic Web. In IN PROCEEDINGS OF THE SECOND INTERNATIONAL SEMANTIC WEB CONFERENCE, 351368,2003

[21] Zaihrayeu, Ilya, Paulo Pinheiro Da Silva, Deborah L. Mcguinness, Ilya Zaihrayeu, Paulo Pinheiro, Silva Deborah, and L. Mcguinness. IWTrust: Improving User Trust in Answers from the Web. In Proceedings of 3rd International Conference on Trust Management (iTrust2005, 384392. Springer, 2005.

[22] Falcone, Rino, Giovanni Pezzulo, and Cristiano Castelfranchi. A Fuzzy Approach to a Belief-based Trust Computation. In Lecture Notes on Artificial Intelligence, 7386. Springer-Verlag, 2003.

[23] Almenrez, Florina, Andrs Marn, Celeste Campo, and Carlos Garca R. PTM: A Pervasive Trust Management Model for Dynamic Open Environments. In IN: FIRST WORKSHOP ON PERVASIVE SECURITY, PRIVACY AND TRUST PSPT04 IN CONJUNTION WITH MOBIQUITOUS, 2004.

[24] Tang, Wen, Yun-Xiao Ma, and Zhong Chen. Managing Trust in Peerto-Peer Networks. Journal of Digital Information Management 3, no. 2 (June 1, 2005): 58.

[25] Kamvar, Sepandar D., Mario T. Schlosser, and Hector Garcia-Molina The Eigentrust Algorithm for Reputation Management in P2P Networks. In Proceedings of the 12th International Conference on World Wide Web, 640651. WWW 03. New York, NY, USA: ACM, 2003. doi: $10.1145 / 775152.775242$

[26] Levien, R.: Attack resistant trust metrics. Draft Ph.D. Thesis, at U.C. Berkeley.

[27] Golbeck, Jennifer. Computing with Social Trust. Springer, 2008, ISBN 1848003552, 9781848003552.

[28] Gong, Li, R. Needham, and R. Yahalom. Reasoning About Belief in Cryptographic Protocols. In , 1990 IEEE Computer Society Symposium on Research in Security and Privacy, 1990. Proceedings, 234248, 1990. doi:10.1109/RISP.1990.63854.

[29] Josang Audun. "Subjective logic". Book Draft, 2013

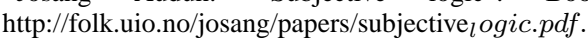

] Zuva, Tranos, Sunday O.Ojo, Seleman Ngwira, and Keneilwe Zuva. A Survey of Recommender Systems Techniques, Challenges and Evaluation Metrics.pdf. International Journal of Emerging Technology and Advanced Engineering, 2012

Massa, P., and P. Avesani. Trust-aware Collaborative Filtering for Recommender Systems. On the Move to Meaningful Internet Systems 2004: CoopIS, DOA, and ODBASE (2004): 492508.

] Haydar, Charif, Anne Boyer, and Azim Roussanaly. Hybridising collaborative filtering and trust-aware recommender systems. In 8th International Conference on Web Information Systems and Technologies -WEBIST2012. Porto, Portugal, 2012. http://hal.inria.fr/hal-00679233.

33] Liu, Xin, and Anwitaman Datta. 2012. Modeling Context Aware Dynamic Trust Using Hidden Markov Model. In Twenty-Sixth AAAI Conference on Artificial Intelligence. 